\title{
COMPOSITE SPECTRA FROM THE FIRST BRIGHT QUASAR SURVEY
}

\author{
M. S. Brotherton ${ }^{1,2}$, Hien D. Tran ${ }^{2,3}$, R. H. Becker ${ }^{2,4}$, Michael D. GregG ${ }^{2,4}$, \\ S. A. Laurent-Muehleisen ${ }^{2,4}$, R. L. White ${ }^{5}$ \\ Draft version October 28, 2018
}

\begin{abstract}
We present a very high signal-to-noise ratio composite spectrum created using 657 radio-selected quasars from the FIRST Bright Quasar Survey. The spectrum spans rest-frame wavelengths $900-$ $7500 \AA$. Additionally we present composite spectra formed from subsets of the total data set in order to investigate the spectral dependence on radio loudness and the presence of broad absorption. In particular, radio-loud quasars are red compared to radio-quiet quasars, and quasars showing low-ionization broad absorption lines are red compared to other quasars. We compare our composites with those from the Large Bright Quasar Survey. Composite quasar spectra have proven to be valuable tools for a host of applications, and in that spirit we make these publically available via the FIRST survey web page.
\end{abstract}

Subject headings: quasars: emission lines, quasars: general

\section{INTRODUCTION}

For over three decades, the construction of composite spectra has proven to be a valuable activity in AGN research. The synthesis of broad-band colors of a large number of quasars produced low-resolution "compromise composite" spectra, enabling the average quasar continuum to be studied over a large wavelength range and $k$-corrections to be computed (Sandage 1966; Evans \& Hart 1977). Emission-line intensity ratios for a large number of quasars over a range of redshifts were combined to determine a composite quasar "spectrum" (Chan \& Burbidge 1975), and it was this technique that first identified the low $\mathrm{L} \alpha / \mathrm{H} \beta$ ratio that posed a problem for photoionization models (Baldwin 1977). A similar bootstrap method established the average emission-line velocity shifts in quasars (Tytler \& Fan 1992).

Technological advances led to large samples of quasars with readily manipulated digital data, resulting in myriad composite spectra in the 1990s. These included composite quasar spectra presented by Boyle (1990), Cristiani \& Vio (1990), and Francis et al. (1991 - using the LBQS, i.e. the Large Bright Quasar Survey), resulting in identification of many weak emission lines and additional recognition of the significance of the omnipresence of Fe II emission blends throughout the ultraviolet and optical. Zheng et al. (1997) produced a composite spectrum using quasar spectra from the Hubble Space Telescope with unprecedented coverage of short wavelengths. These composites have been used extensively for many applications.

Composite spectra also permit investigation of the spectral dependencies on other properties. Sprayberry \& Foltz (1992) compared the composite spectra of quasars with broad absorption lines (BALs) to those without, finding that quasars with low-ionization BALs have significantly redder continua consistent with line-of-sight dust. Fran- cis, Hooper, \& Impey (1993), using the LBQS data set, created composite spectra of radio-loud and radio-quiet quasars separately, finding an extra low-velocity emission line component in the former (also see Cristiani \& Vio 1990 and Zheng et al. 1997). Brotherton et al. (1994) created composites from two samples of quasars distinguished by the velocity width of the broad lines, finding significant differences in the line ratios of the two. Green (1998) created two composites distinguished by X-ray brightness, finding in particular X-ray bright quasars had significantly stronger narrow-line emission. Baker \& Hunstead (1995) created composites using samples distinguished by their radio structure, finding that radio-loud quasars that appear to be more edge-on also appear to be dustier. Malhotra (1997) created a composite spectrum by aligning the redshifts of intervening $\mathrm{Mg}$ II absorbers present in quasar spectra and detected the signature of the $2175 \AA$ feature associated with dust from the absorbers.

In this paper, we present a very high-quality composite spectrum constructed using 657 quasars from the FIRST Bright Quasar Survey (FBQS) (White et al. 2000), as well as several composite spectra formed using subsamples of the FBQS. Section 2 describes the sample selection and data set used to construct the composite spectra, as well as the algorithm we use to construct composite spectra. Section 3 describes the composite spectra themselves. Section 4 discusses the properties of the FBQS we can discern from the composite spectra and compares our results with those of the Large Bright Quasar Survey (hereafter LBQS) (Foltz et al. 1987, 1989; Hewett et al. 1991; Chaffee et al. 1991; Morris et al. 1991; Francis et al. 1991; Francis et al. 1992). Section 5 summarizes our results.

\section{SAMPLE AND DATA}

${ }^{1}$ KPNO/NOAO, 950 N. Cherry Ave., P. O. Box 26732, Tucson, AZ 85726

${ }^{2}$ Institute of Geophysics and Planetary Physics, Lawrence Livermore National Laboratory, 7000 East Avenue, P.O. Box 808, L413, Livermore, CA 94550

${ }^{3}$ Current Address: Johns Hopkins University, Baltimore, MD 21218

${ }^{4}$ Physics Dept., University of California-Davis

${ }^{5}$ Space Telescope Science Institute, 3700 San Martin Dr., Baltimore, MD 21218 
The FBQS starts with radio sources found using the NRAO $^{6}$ Very Large Array FIRST survey (Becker et al. $1995)$, a $20 \mathrm{~cm}$ survey at $\sim 5^{\prime \prime}$ resolution with a detection limit $\sim 1 \mathrm{mJy}$. Quasar candidates are determined by matching FIRST sources within 1".2 of stellar sources with $O-E<2.0$ and $E<17.8$ from the Automated Plate Measuring Facility (APM) catalog of the Palomar Observatory Sky Survey I (POSS-I) plates (MacMahon et al. 2000). The choice of selection criteria yields quasars and BL Lac objects with $60 \%$ selection efficiency and misses very few bright red quasars (Gregg et al. 1996 - the pilot investigation). White et al. (2000) presents the second installment of the FBQS with a catalog of 636 quasars distributed over $2682 \mathrm{deg}^{2}$, fully characterizing the sample and displaying nearly all the spectra we use to construct our composites. The spectra are a heterogenous set, coming from a half dozen telescopes, although generally have $\sim 10 \AA$ resolution and signal-to-noise ratios of 20 or higher. Both radio-loud and radio-quiet quasars are present in approximately equal numbers, as are radio-loud BAL quasars (Becker et al. 2000), and large numbers of radio-intermediate quasars. This is the first radio-selected sample that is competitive in size with optically selected quasar surveys such as the LBQS.

\section{COMPOSITE SPECTRA}

\subsection{Methodology and Caveats}

In constructing our composite spectra, we use essentially the same algorithm described by Francis et al. (1991) which they used to construct their LBQS composite quasar spectrum. In fact, we employ the same code that has been used to construct the composite spectrum of the total LBQS sample (S. Morris 1999, private communication), with which we make a comparison in $\S 4$. Below we describe the procedure, which employs IRAF except as noted.

The spectra are rebinned to the same wavelength range, $3200 \AA-9200 \AA$. Zeroes are used for regions within this range with no data (these zero-padded regions are given zero weight in the combination). These spectra are then stacked into a single image. A similar procedure is followed to produce a stacked image of noise spectra.

Noise spectra are not available for all the spectra in the FBQS database and we have used a single uniform method to estimate the noise for all spectra which will be used to weight individual spectra in the composite. A high-order continuum fit is used to normalize each spectrum. We then use a VISTA script to measure the average $\mathrm{S} / \mathrm{N}$ from these. The spectra are smoothed by 9 pixels, their square root is taken, then they are multiplied by the average $\mathrm{S} / \mathrm{N}$ which provides an estimate of the true $\mathrm{S} / \mathrm{N}$ spectrum. The $\mathrm{S} / \mathrm{N}$ is set to 1 across the atmospheric $\mathrm{A}$ and $\mathrm{B}$ bands to ensure these regions receive low weight in the combination. The spectra are weighted by $(\mathrm{S} / \mathrm{N})^{2}$ when combined.

A separate file is also prepared that contains the redshifts of the input spectra, determined by using crosscorrelation against a preliminary composite spectrum and refined in an iterative manner. The final composite spectrum clearly resolves the [O III $] \lambda \lambda 4959,5007$ narrow lines (each with a FWHM of $750 \mathrm{~km} \mathrm{~s}^{-1}$ ), while initial versions of the composite using visually assigned redshifts showed broader lines that were somewhat blended. Our success with using cross-correlation redshifts mirrors the experience of Francis et al. (1991) in constructing the LBQS composite.

The flux and noise spectral images are stacked into a single three-dimensional data cube, which is the input for the IRAF procedure provided by Simon Morris. The procedure uses the algorithm described by Francis et al. (1991). Spectra are added, from low- $z$ to high- $z$, to a running average. All spectra are rebinned to a common dispersion. The squares of the signal-to-noise ratios are used to weight the individual spectra. New spectra are normalized to the running average in the region of overlap only. A standard deviation is computed.

As discussed by Francis et al. (1991), such a procedure has a number of limitations and uncertainties. All of the caveats that applied there also apply to the FBQS composite. Galactic and intrinsic extinction may artificially redden individual spectra, although the FBQS is selected from objects at high Galactic latitude and a (mild) color criterion is imposed. Slit losses from differential refraction may redden individual spectra, although the majority of spectra were obtained at Lick and Keck Observatories where the spectrograph slits were always at the parallactic angle. The appearance of features in the composite spanning large wavelengths (e.g., the overall continuum shape and the $3000 \AA$ "little blue bump") is sensitive to the order spectra are added to the composite; in the LBQS, Francis et al. (1991) reported that the spectral index $\alpha$ varied by \pm 0.2 , which is consistent with our own tests we discuss in $\S 3.2$. In a flux-limited sample such as the FBQS or LBQS, quasars at low $z$ are typically lower luminosity than those found at high $z$ (see Francis et al. 1991 and White et al. 2000 for illustrative plots), and spectral dependencies on luminosity (e.g., Korista et al. 1998) may lead to spurious results in comparing optical to ultraviolet properties. Finally, the arithmetic mean of power-law spectra is not necessarily a power-law spectrum with the mean power-law index.

Below we characterize the total FBQS composite spectrum quantitatively in some detail. We provide less detail for the composites formed from the subsamples based on radio and absorption properties; because of the above caveats the results of our comparisons should be regarded as suggestive but not conclusive without follow-up investigations. These composite spectra are useful tools and a powerful way to graphically illustrate certain dependencies, but the reader is cautioned not to overinterpret these results.

\subsection{FBQS Composite Quasar Spectrum}

About $90 \%$ of the 657 spectra used in construction of the FBQS composite are plotted by White et al. (2000) and additional details may be found there. Confirmed and probable BAL quasars have been excluded from the composite. Figure 1 plots the composite spectrum and labels major emission lines. Figure 2 plots the histogram of the number of quasars contributing at each wavelength. Figure 3 plots the standard deviation spectrum; it differs from

\footnotetext{
${ }^{6}$ The National Radio Astronomy Observatory is a facility of the National Science Foundation operated under cooperative agreement by Associated Universities, Inc..
} 
the mean spectrum most noticeably in the strength of the narrow lines, particularly [O III] $\lambda \lambda 4959,5007$. The signalto-noise ratio per $0.6 \AA$ pixel peaks at just under 200 (estimated by using the standard deviation spectrum and the number of objects contributing to each pixel to calculate the standard error in the mean); rebinning to $2.5 \AA$ increases the signal-to-noise ratio to just under 400. Figure 4 plots the composite in log-log space in which a powerlaw is represented as a straight line; a power-law with a spectral index $\alpha=-0.46\left(\mathrm{~F}_{\nu} \propto \nu^{\alpha}\right)$ appears to be a good estimate of an underlying continuum between $\mathrm{H} \beta$ and $\mathrm{L} \alpha$.

Table 1 lists emission features present in the spectrum along with measurements of line ratios and equivalent widths. The choices of integration windows are made to match those of Francis et al. (1991) and to facilitate comparison to the LBQS composite spectrum (§ 4). In making measurements of the standard deviation of equivalent widths it is assumed that the continuum and line flux vary independently.

In order to investigate to what extent the spectral energy distribution may be a function of the normalization process, we also constructed a composite beginning with the largest redshift quasars and working backwards to the lowest redshift quasars, the reverse of the procedure used to construct the spectrum in Figure 1. The result was a very similar but slightly redder spectrum, also well fit by a power-law but with a spectral index $\alpha=-0.49$ (which gives rise to a $12 \%$ difference at $\mathrm{L} \alpha$ vs. $\mathrm{H} \beta$ ); the emission-lines are identical between the two and divide out as expected.

\subsection{Composites with Different Radio-Loudness}

We can categorize the FBQS quasars according to their radio-loudness. As recommended by Weymann (1997), we adopt $\log \mathrm{R}^{*}$ as the formal measure of radio-loudness with $\log \mathrm{R}^{*}=1.0$ dividing radio-loud and radio-quiet subsamples, where $\mathrm{R}^{*}$ is the $K$-corrected ratio of radio-to-optical power (Sramek \& Weedman 1980; Stocke et al. 1992). Log $\mathrm{R}^{*}$ is tabulated for the FBQS in White et al. (2000). The FIRST quasars, while radio-selected, in fact include a large fraction of radio-quiet quasars. Using the $\mathrm{R}^{*}$ criterion, our data set contains $59 \%$ radio-loud quasars with redshifts up to $z=3.4$ and $41 \%$ radio-quiet quasars with redshifts up to $z=3.3$ (see Fig. 14 of White et al. 2000). The range in optical luminosities is also quite similar, although there exist significant differences in the luminosity ranges compared at low $z$ vs. high $z$ that result from the FBQS being a magnitude-limited sample. Figure 5 plots the radio-loud and radio-quiet quasar composite spectra.

Compared to the radio-quiet composite, the radioloud spectrum has a redder spectral energy distribution, broader Balmer lines, stronger [O III] emission, and a stronger red wing/weaker blue wing asymmetry to the C IV $\lambda 1549$ emission line profile. Similar differences have been previously noted (e.g., Boroson \& Green 1992; Barthel, Tytler, \& Thomson 1990).

We also experimented with three divisions of radioloudness, such that radio-intermediate quasars were distinguished as those with $0.5<\log \mathrm{R}^{*}<1.5$. The FBQS is sensitive to radio-intermediate quasars, which are notably missing from earlier searches (White et al. 2000). The distribution of "radio-loudness" is not significantly bimodal. The radio-intermediate quasar composite spectrum closely resembles that of the radio-loud quasars, and so differs from the radio-quiet quasar composite spectrum in a very similar manner.

\subsection{BAL Quasar Composites}

We have constructed composite spectra of samples of broad absorption line (BAL) quasars in the FBQS. These samples include high-ionization BAL quasars ( 25 objects) and the low-ionization BAL quasars (18 objects, including 4 metastable $\mathrm{Fe}$ absorbed quasars). The majority of objects are cataloged and characterized by Becker et al. (2000) (29 objects, 15 high-ionization BAL quasars and 14 low-ionization BAL quasars); in order to increase our sample sizes and the significance of the results, we used all the BAL quasars in the FBQS database whether published or not. Note that we have erred on the side of inclusivity, as discussed by Becker et al. (2000), and included several confirmed and probable BAL quasars that do not meet the criterion of positive BALnicity index (Weymann et al. 1991), e.g. FIRST J1603+3002, which has been shown to have an intrinsic high-velocity outflow (Arav et al. 1999). Figure 6 plots these composite spectra as well as the total FBQS composite.

The colors of the low-ionization BAL quasar composite are redder than those of the high-ionization BAL quasar composite, which are in turn redder than that of the FBQS composite. The continuum band colors of Yamamoto \& Vansevicius (1999) indicate that the BAL quasar composites are consistent with reddening the FBQS composite according to a Small Magallenic Cloud (SMC) extinction law. If we assume such a law, the spectral shape of the high-ionization BAL quasar composite is consistent with the FBQS composite if dereddened by $E(B-V) \sim 0.04$ mag, while the low-ionization BAL quasar composite must be dereddened by $E(B-V) \sim 0.1 \mathrm{mag}$. Small color differences in the rest-frame optical such as these become magnified in the rest-frame ultraviolet as seen in the figure.

BALs are not so readily apparent in the high-ionization BAL quasar composite spectrum. This results from a wide range in BAL properties, primarily the velocity offsets from the systemic redshift, coupled with a paucity of quasars with extremely deep and broad troughs (e.g., as seen in PHL 5200). The BAL features are therefore diminished in the averaging process.

\section{DISCUSSION}

We restrict much of our discussion to a comparison between our FBQS composite spectra and similar ones created from the LBQS data set. This is appropriate because of the similarities between the surveys in terms of luminosities, redshift range, and size. This is also appropriate as composites from the LBQS have been extensively used for a decade, and include composites made from subsamples with BALs and with differing degrees of radio loudness. Finally, we can compare our FBQS composite quasar spectrum to a LBQS composite quasar spectrum using exactly the same algorithm and software. Table 2 summarizes the FBQS and LBQS samples explored here with composite spectra, and a few of the properties of the composite spectra.

The LBQS composite spectrum of Francis et al. (1991) was created using 688 of the total LBQS sample of 1018 
quasars, or $68 \%$. Following the completion of the LBQS a composite spectrum was created using the total data set and has been made available to us along with the software used to create it (S. Morris 1999, private communication). The properties of the last $\sim 300$ quasars are consistent with those which went into the first composite, but the two composites differ significantly in a number of ways. The source of the differences is not entirely clear although it has been speculated that the difference has to do with the vagaries of the normalization procedure (P. Francis 1999 , private communication).

Figure 7 compares the LBQS composite quasar spectra of Francis et al. (1991) and Morris (1999, private communication). It is readily apparent that the shape of the spectral energy distributions differ, with $10 \%$ excursions in the optical and near-UV, to a $20 \%$ difference in the far-UV (keeping in mind the presence of the Lyman $\alpha$ forest that depresses the intrinsic quasar continuum at these wavelengths). The original LBQS survey spectra suffered from differential atmospheric slit losses, and the degree of corrections made or not made to the spectra at the time of the construction of the composite spectra likely contribute to the changes in the large scale continuum shape (P. Francis 2000 , private communication).

The ultraviolet emission lines also differ between the LBQS composite spectra. Notably, the $\mathrm{L} \alpha, \mathrm{N} \mathrm{V}$ blend is $25 \%$ stronger in the new composite, and the C IV $\lambda 1549$ line is $10 \%$ stronger and may have a rather different profile. These differences should be kept in mind when making comparisons to tabulated line ratios and equivalent widths in Francis et al. (1991). The weakness of the $\mathrm{L} \alpha$ and $\mathrm{C}$ IV $\lambda 1549$ lines in the Francis et al. (1991) composite may result from the inclusion of objects with strong associated absorption or weak BALs, which are probably appropriate to include for the purpose of making $k$-corrections but compromise measuring accurate intrinsic average line ratios.

In comparing the FBQS composite quasar spectrum to that of the LBQS, the different biases in sample selection and the ensuing sample differences should first be made clear. The LBQS used purely optical techniques to select candidate quasars, resulting in a very different distribution of radio-loudness in that sample (approximately 10\% radio-loud quasars - see Hooper et al. 1995). While about half the FBQS quasars are formally radio-quiet, the more extreme radio-quiets and all radio-silent quasars are not represented, especially at high redshift. The magnitude range of the LBQS $\left(16.0 \leq m_{B_{J}} \leq 18.85\right)$ is different from that of the FBQS $(E \leq 17.8)$. The inclusion of very bright sources in the FBQS may explain why its distribution of redshifts includes a larger fraction of low- $z$ objects compared to the LBQS; the redshift ranges are similar.

The overall spectral shape of the FBQS composite spectrum is more similar to that of the total LBQS composite spectrum than that of Francis et al. (1991). The FBQS spectrum is slightly redder. It may be that quasar spectra from the rest-frame ultraviolet through the optical can be better represented by single power laws than suggested by Francis et al. (1991), but this should be investigated more thoroughly by examining spectrophotometry of individual objects with wavelength coverage spanning from Lyman $\alpha$ through the Balmer lines. The FBQS spectrum also has stronger Lyman $\alpha$ and [O III] emission, and the
C IV profile has a stronger red wing than blue wing. The differences in the $\mathrm{C}$ IV profile and [O III] emission are also seen in Figure 5, the comparison between the radio-loud and radio-quiet subsamples of the FBQS.

Figure 5 fails to show a difference in Lyman $\alpha$, however. Francis, Hooper, \& Impey (1993) created composite ultraviolet spectra of radio-quiet and radio-loud subsamples of the LBQS. These spectra differed at a marginally statistically significant level, in the sense that radio-loud quasars had stronger low-to-intermediate velocity emission, primarily from C IV and Lyman $\alpha$. The FBQS may not be sampling sufficient numbers of quasars, nor a sufficient range in radio-loudness. The fraction of radio-quiet quasars containing the Lyman $\alpha$ emission line is only $25 \%$ (out of 50 quasars with spectra that blue, cf. $39 \%$ with $\log \mathrm{R}^{*}<1$ in the FBQS as a whole), limiting our statistics. These highest redshift radio-quiet quasars also possess the largest $\log \mathrm{R}^{*}$ values of the radio-quiet class, and as radio loudness appears to be a more continuous property than once thought (White et al. 2000), it may not be unexpected that Lyman $\alpha$ is so similar in the radio-loud and radio-quiet subsamples of the FBQS.

That the FBQS composite has an unusually strong Lyman $\alpha$ cannot be denied. Cannonical values of C IV/Ly $\alpha$ lie within the range $0.4--0.6$ (Baldwin et al. 1995), although most of these are drawn from optically selected samples. Using an estimate of $25 \%$ contamination from $\mathrm{N}$ $\mathrm{V}$ in the Lyman $\alpha$ blend, the FBQS composite spectrum shows C IV $/$ Ly $\alpha=0.36$, just outside the cannonical range. The C IV/(Ly $\alpha+\mathrm{N}$ V) ratio from Francis et al. (1991) is 0.63 , but the ratio in the updated LBQS composite is only 0.37 , or 0.47 for $\mathrm{C}$ IV/Ly $\alpha$ after correcting for an estimated $25 \% \mathrm{~N} \mathrm{~V}$ contamination. That the Lyman $\alpha$ emission line is stronger in the FBQS than in the LBQS is not simply a matter of absorbed quasars.

This difference is probably related to the presence of strong extended narrow-line regions/nebulosity seen preferentially in radio-loud objects (e.g., Heckman et al. 1991). Very large Ly $\alpha / \mathrm{C}$ IV ratios are seen in high-redshift radio galaxies (e.g., McCarthy 1993) and the narrow-line regions of Seyfert 2 galaxies (e.g., Ferland \& Osterbrock 1986). Narrow-line emission in general is stronger in radioloud quasars (Boroson \& Green 1992). These differences are tied into the so-called "eigenvector 1" relationships of principal component analysis (e.g., Boroson \& Green 1992; Francis et al. 1992; Brotherton et al. 1994; Wills et al. 1999), which places radio-loud quasars with strong narrow and intermediate emission lines at one extreme, and radio-quiet quasars and narrow-line Seyfert 1 galaxies at the other. The current prevailing explanation for at least a portion of these trends involves the variation in accretion rate and covering fraction (e.g., Boroson \& Green 1992).

Sprayberry \& Foltz (1992) examined composite spectra of BAL quasar subsamples of the LBQS (see also Weymann et al. 1991). They concluded that the spectral shape of the composite low-ionization BAL quasar composite spectrum was consistent with that of the highionization BAL quasar composite spectrum if reddened by an SMC-type extinction law with $E(B-V) \sim 0.1$. This is rather similar to what we find for the FBQS, an entirely consistent result given the small sample sizes and normalization uncertainties. There remains the question of just how many low-ionization BAL quasars were lost 
from the sample because of the magnitude limit; their existence will probably make the true average low-ionization BAL quasar more reddened than this.

\section{SUMMARY}

We have created a composite quasar spectrum of the total non-BAL FBQS sample and additional subsamples of particular interest, including radio-quiet, radio-loud, and high and low ionization BAL quasars. The composite spectra are publically available via the FIRST Survey web page ${ }^{7}$. The process used to create the composites is identical to that used in creating the composite spectra of the LBQS (Francis et al. 1991; S. Morris 1999, private communication), to which we have compared our FBQS composite spectra. While as robust as possible, there are unavoidable uncertainties inherent in the procedure of creating composite spectra, and these uncertainties should be considered when using such spectra for particular applications.
We thank Simon Morris, Kirk Korista, and Paul Francis for making available their software and LBQS composite spectra, as well as for additional commentary on this manuscript and the originally published LBQS composite spectrum. We thank everyone who has contributed or is continuing to contribute to the FIRST Bright Quasar Survey. The success of the FIRST survey is in large measure due to the generous support of a number of organizations. In particular, we acknowledge support from the NRAO, the NSF (grants AST-98-02791 and AST-98-02732), the Institute of Geophysics and Planetary Physics (operated under the auspices of the U.S. Department of Energy by Lawrence Livermore National Laboratory under contract No. W-7405-Eng-48), the Space Telescope Science Institute, NATO, the National Geographic Society (grant NGS No. 5393-094), Columbia University, and Sun Microsystems.

\section{REFERENCES}

Arav, N., Becker, R. H., Laurent-Muehleisen, S. A., Gregg, M. D., White, R. L., Brotherton, M. S. and de Kool, M. 1999, ApJ, 524, 566

Baker, J. C. \& Hunstead, R. W. 1995, ApJ, 452, L95

Baldwin, J. A. 1977, MNRAS, 178, 67P

Baldwin, J., Ferland, G., Korista, K. and Verner, D. 1995, ApJ, 455, L119

Barthel, P. D., Tytler, D. R. and Thomson, B. 1990, A\&AS, 82, 339

Becker, R. H., White, R. L., \& Helfand, D. J. 1995, ApJ, 450, 559

Boroson, T. A., \& Green, R. F. 1992, ApJS, 80, 109

Boyle, B. J. 1990, MNRAS, 243, 231

Brotherton, M. S., Wills, B. J., Francis, P. J. \& Steidel, C. C. 1994, ApJ, 430, 495

Chaffee, F. H., Foltz, C. B., Hewett, P. C., Francis, P. J., Weymann, R. J., Morris, S. L., Anderson, S. F., \& MacAlpine, G. M. 1991, AJ, 102, 461

Chan, Y. -W. T. \& Burbidge 1975 \& Burbidge, E. M. 1975, ApJ, 198,45

Cristiani, S. \& Vio, R. 1990, A\&A, 227, 385

Evans, A. \& Hart, D. 1977, A\&A, 58, 241

Ferland, G. J. and Osterbrock, D. E. 1986, ApJ, 300, 658

Foltz, C. B., Chaffee, F. H., Hewett, P. C., MacAlpine, G. M., Turnshek, D. A., Weymann, R. J., \& Anderson, S. F. 1987, AJ, 94, 1423

Foltz, C. B., Chaffee, F. H., Hewett, P. C., Weymann, R. J., Anderson, S. F., \& MacAlpine, G. M. 1989, AJ, 98, 1959

Francis, P. J., Hewitt, P. C., Foltz, C. B., \& Chaffee, F. H. 1992, ApJ, 398, 476

Francis, P. J., Hewett, P. C., Foltz, C. B., Chaffee, F. H., Weymann, R. J. \& Morris, S. L. 1991, ApJ, 373, 465

Francis, P. J., Hooper, E. J. \& Impey, C. D. 1993, AJ, 106, 417

Green, P. J. 1998, ApJ, 498, 170
Gregg, M. D., Becker, R. H., White, R. L., Helfand, D. J., McMahon, R. G., \& Hook, I. M. 1996, AJ, 112, 407

Heckman, T. M., Lehnert, M. D., Miley, G. K. and van Breugel, W. 1991, ApJ, 381, 373

Hewett, P. C., Foltz, C. B., Chaffee, F. H., Francis, P. J., Weymann, R. J., Morris, S. L., Anderson, S. F., \& MacAlpine, G. M. 1991, AJ, 101, 1121

Hooper, E. J., Impey, C. D., Foltz, C. B. and Hewett, P. C. 1995, ApJ, 445, 62

Korista, K., Baldwin, J. and Ferland, G. 1998, ApJ, 507, 24

Malhotra, S. 1997, ApJ, 488, L101

McCarthy, P. J. 1993, ARA\&A, 31, 639

Morris, S. L., Weymann, R. J., Anderson, S. F., Hewett, P. C., Chaffee, F. H., Francis, P. J., \& MacAlpine, G. M. 1991, AJ, 102, 1627

Sandage, A. 1966, ApJ, 146, 13

Sprayberry, D. \& Foltz, C. B. 1992, ApJ, 390, 39

Sramek, R., \& Weedman, D. 1980, ApJ. 238, 435

Stocke, J. T., Morris, S. L., Weymann, R. J., \& Foltz, C. B. 1992, ApJ, 396, 487

Tytler, D., \& Fan, X. 1992, ApJS, 79, 1

Weymann, R. 1997, ASP Conf. Ser. 128: Mass Ejection from Active Galactic Nuclei, 3

Weymann, R. J., Morris, S. L., Foltz, C. B. and Hewett, P. C. 1991, ApJ, 373, 23

White, R. L., Becker, R. H., Helfand, D. J., \& Gregg, M. D. 1997, ApJ, 475, 479

White, R. L. and 13 colleagues 2000, ApJS, 126, 133

Wills, B. J., Laor, A., Brotherton, M. S., Wills, D., Wilkes, B. J., Ferland, G. J. and Shang, Z. 1999, ApJ, 515, L53

Yamamoto, T. M. and Vansevivcius, V. 1999, PASJ, 51, 405

Zheng, W. , Kriss, G. A., Telfer, R. C., Grimes, J. P. \& Davidsen, A. F. 1997, ApJ, 475, 469 
TABLE 1

Line StRengths

\begin{tabular}{|c|c|c|c|c|c|c|}
\hline Emission Feature & $\begin{array}{c}\text { Rest } \lambda \\
(\AA)\end{array}$ & $\begin{array}{c}\text { Start }^{\mathrm{a}} \\
(\AA)\end{array}$ & $\begin{array}{l}\operatorname{End}^{\mathrm{a}} \\
(\AA)\end{array}$ & Intensity $^{\mathrm{b}}$ & $\sigma^{\mathrm{c}}$ & $\begin{array}{c}\mathrm{EW}_{\text {rest }} \\
(\AA)\end{array}$ \\
\hline $\mathrm{Ly} \beta+\mathrm{O}$ VI & $1026 \& 1034$ & 1018 & 1054 & 11.6 & $\ldots$ & 11 \\
\hline $\mathrm{Ly} \alpha+\mathrm{N} \mathrm{V}$ & $1216 \& 1240$ & 1186 & 1286 & 100 & 30 & 87 \\
\hline O I & 1302 & 1288 & 1325 & 2.4 & $\ldots$ & 2.3 \\
\hline C II & 1335 & 1325 & 1354 & 0.7 & $\ldots$ & 0.7 \\
\hline $\mathrm{Si}$ IV + O IV] & 1400 & 1353 & 1454 & 6.8 & 1.1 & 7.3 \\
\hline C IV & 1549 & 1452 & 1602 & 27 & 11 & 33 \\
\hline He II + O III] & $1640 \& 1663$ & 1602 & 1700 & 5.2 & 0.4 & 7.0 \\
\hline $\mathrm{Al} \mathrm{III} \mathrm{+} \mathrm{C} \mathrm{III}]$ & $1858 \& 1909$ & 1828 & 1976 & 10 & 2.9 & 17 \\
\hline 2000 feature & $\ldots$ & 1985 & 2018 & 0.2 & $\ldots$ & 0.4 \\
\hline 2080 feature & $\ldots$ & 2035 & 2125 & 1.2 & $\ldots$ & 2.2 \\
\hline C II] & 2326 & 2242 & 2388 & 1.4 & $\ldots$ & 3.0 \\
\hline$[\mathrm{Ne} I V]$ & 2423 & 2386 & 2464 & 0.4 & $\ldots$ & 0.8 \\
\hline Mg II & 2798 & 2650 & 2916 & 13 & 4.4 & 34 \\
\hline 2970 feature & $\ldots$ & 2908 & 3026 & 1.3 & $\ldots$ & 3.7 \\
\hline 3130 feature & $\ldots$ & 3100 & 3156 & 0.2 & $\ldots$ & 0.8 \\
\hline 3200 feature & $\ldots$ & 3156 & 3236 & 0.3 & $\ldots$ & 1.1 \\
\hline$[\mathrm{Ne} \mathrm{V}]$ & 3346 & 3324 & 3372 & 0.1 & $\ldots$ & 0.3 \\
\hline$[\mathrm{Ne} \mathrm{V}]$ & 3426 & 3392 & 3452 & 0.4 & $\ldots$ & 1.5 \\
\hline$\left[\begin{array}{ll}\mathrm{O} & \mathrm{II}\end{array}\right]$ & 3727 & 3712 & 3742 & 0.6 & 0.6 & 2.6 \\
\hline$[\mathrm{Ne} \mathrm{III}]+\mathrm{He} \mathrm{I}$ & $3869 \& 3889$ & 3804 & 3934 & 1.1 & $\ldots$ & 5.5 \\
\hline$[\mathrm{Ne}$ III] & 3968 & 3934 & 4012 & 0.4 & $\ldots$ & 2.2 \\
\hline$[\mathrm{S} \mathrm{II}]+\mathrm{H} \delta$ & $4068 / 4076$ \& 4102 & 4044 & 4148 & 0.8 & $\ldots$ & 4.4 \\
\hline $\mathrm{H} \gamma+[\mathrm{O}$ III] & $4340 \& 4363$ & 4276 & 4405 & 2.8 & 0.1 & 12 \\
\hline $\mathrm{H} \beta$ & 4861 & 4704 & 5112 & 10 & 2.8 & 75 \\
\hline [O III] & 4959 & 4942 & 4976 & 1.2 & 1.3 & 7.5 \\
\hline [O III $]$ & 5007 & 4986 & 5044 & 5.3 & 6.5 & 34 \\
\hline $\mathrm{He} \mathrm{I}$ & 5876 & 5743 & 6015 & 1.7 & $\ldots$ & 17 \\
\hline $\mathrm{H} \alpha$ & 6563 & 6400 & 6800 & 31 & 12 & 300 \\
\hline \multicolumn{7}{|c|}{ Fe II COMPONENTS: } \\
\hline 1 & $\ldots$ & 1610 & 2210 & $\ldots$ & $\ldots$ & $\ldots$ \\
\hline 2 & $\ldots$ & 2210 & 2730 & 27 & 5.5 & $\ldots$ \\
\hline $3^{\mathrm{d}}$ & $\ldots$ & 2960 & 4040 & 35 & 2.2 & $\ldots$ \\
\hline 4 & $\ldots$ & 4340 & 4830 & 3.6 & 0.8 & $\ldots$ \\
\hline 5 & $\ldots$ & 5050 & 5520 & 2.0 & 1.1 & $\ldots$ \\
\hline
\end{tabular}

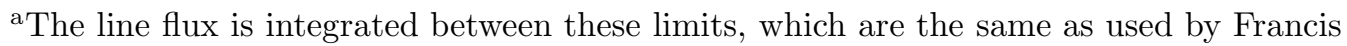
et al. (1991).

${ }^{\mathrm{b}}$ Percent of flux of $\operatorname{Ly} \alpha+\mathrm{N}$ V blend.

${ }^{\mathrm{c}}$ Standard deviation, under the assumption that the line variation is independent of that of the continuum.

${ }^{\mathrm{d}}$ This component includes the Balmer continuum. 
TABLE 2

Summary of Samples \& Select Composite Properties

\begin{tabular}{lcccc}
\hline \hline $\begin{array}{l}\text { Sample (N) } \\
\text { Subsample (\%) }\end{array}$ & Selection & $\langle z>$ & $\begin{array}{c}\mathrm{N}_{\text {peak }}{ }^{\mathrm{a}} \\
(\AA)\end{array}$ & $\alpha^{\mathrm{b}}$ \\
\hline FBQS (657) & FIRST, $E<17.8$ & 1.0 & $\sim 2900$ & -0.46 \\
Radio-loud (61\%) & $\log \mathrm{R}^{*}>1$ & 1.1 & $\sim 2800$ & -0.50 \\
Radio-quiet (39\%) & $\log \mathrm{R}^{*}<1$ & 0.9 & $\sim 3200$ & -0.42 \\
& & & & \\
LBQS (1018) & Optical techniques & 1.3 & $\sim 2200$ & -0.32 \\
Francis et al. 1991 (68\%) & $16.0 \leq m_{B_{J}} \leq 18.85$ & 1.3 & $\sim 2200$ & -0.32 \\
\hline
\end{tabular}

a The approximate wavelength at which the histogram of the number of quasars contributing to the composite spectrum peaks.

${ }^{\text {b }}$ The optical/ultraviolet power-law index fit between $1450 \AA$ and $5050 \AA$, $\mathrm{F}_{\nu} \propto \nu^{\alpha}$. 


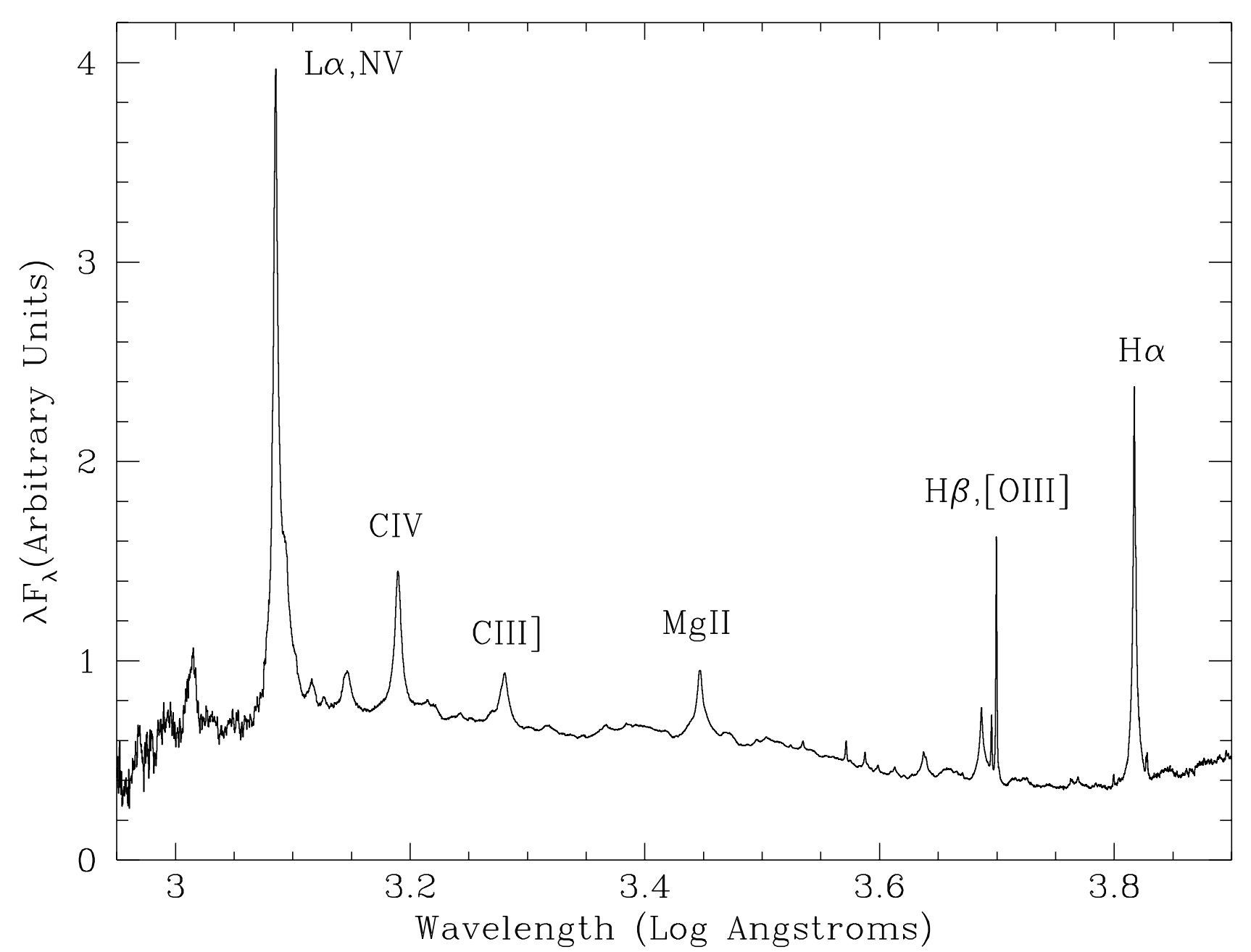

FIG. 1.- FBQS composite spectrum plotted as $\lambda F_{\lambda}$ vs. the logarithm of the rest-frame wavelength. Prominent emission features are marked. The y-axis has been normalized such that the spectrum has values on order of unity. 


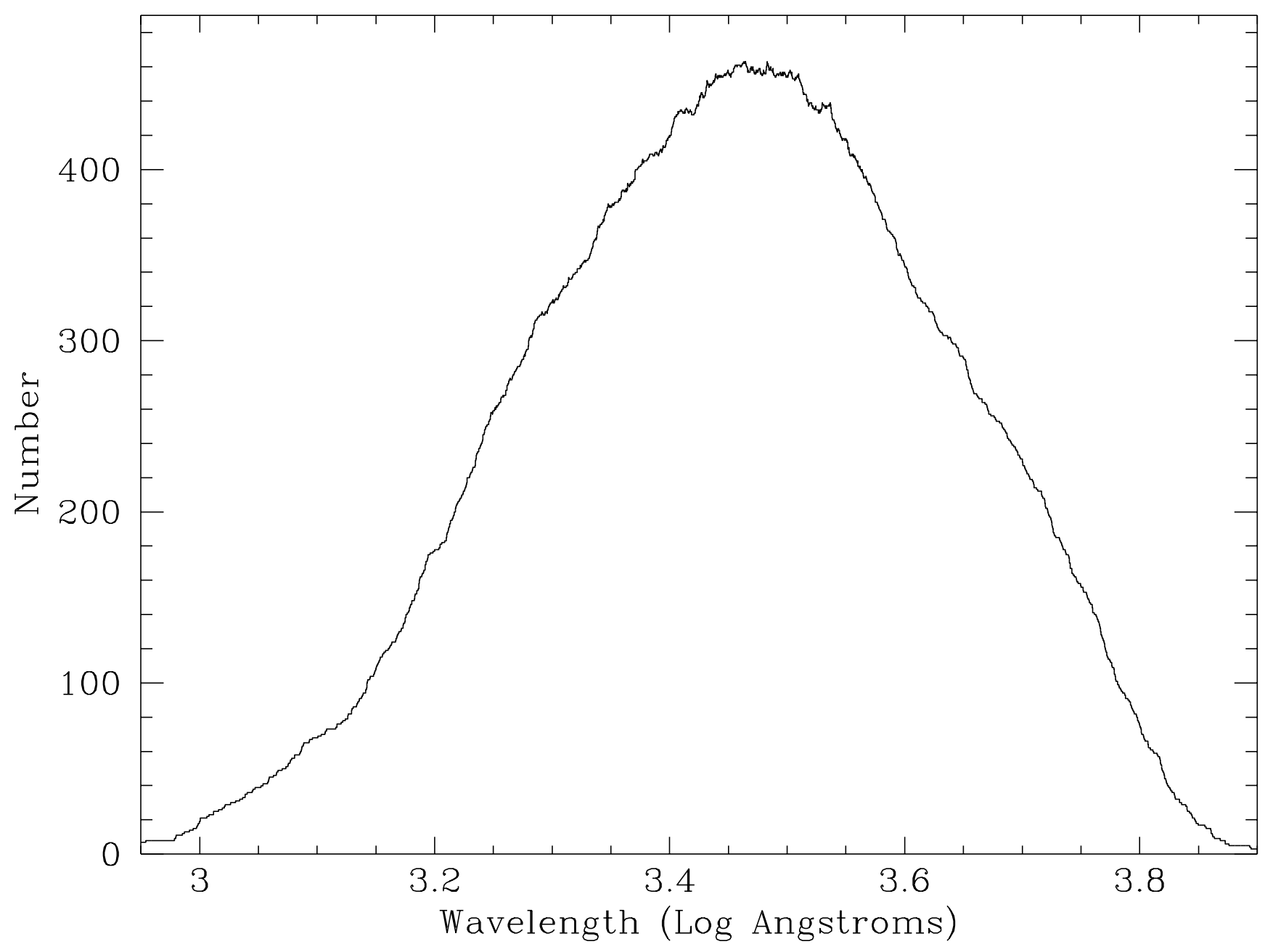

FIG. 2.- Histogram of the number of quasars contributing at each wavelength to the FBQS composite spectrum. 


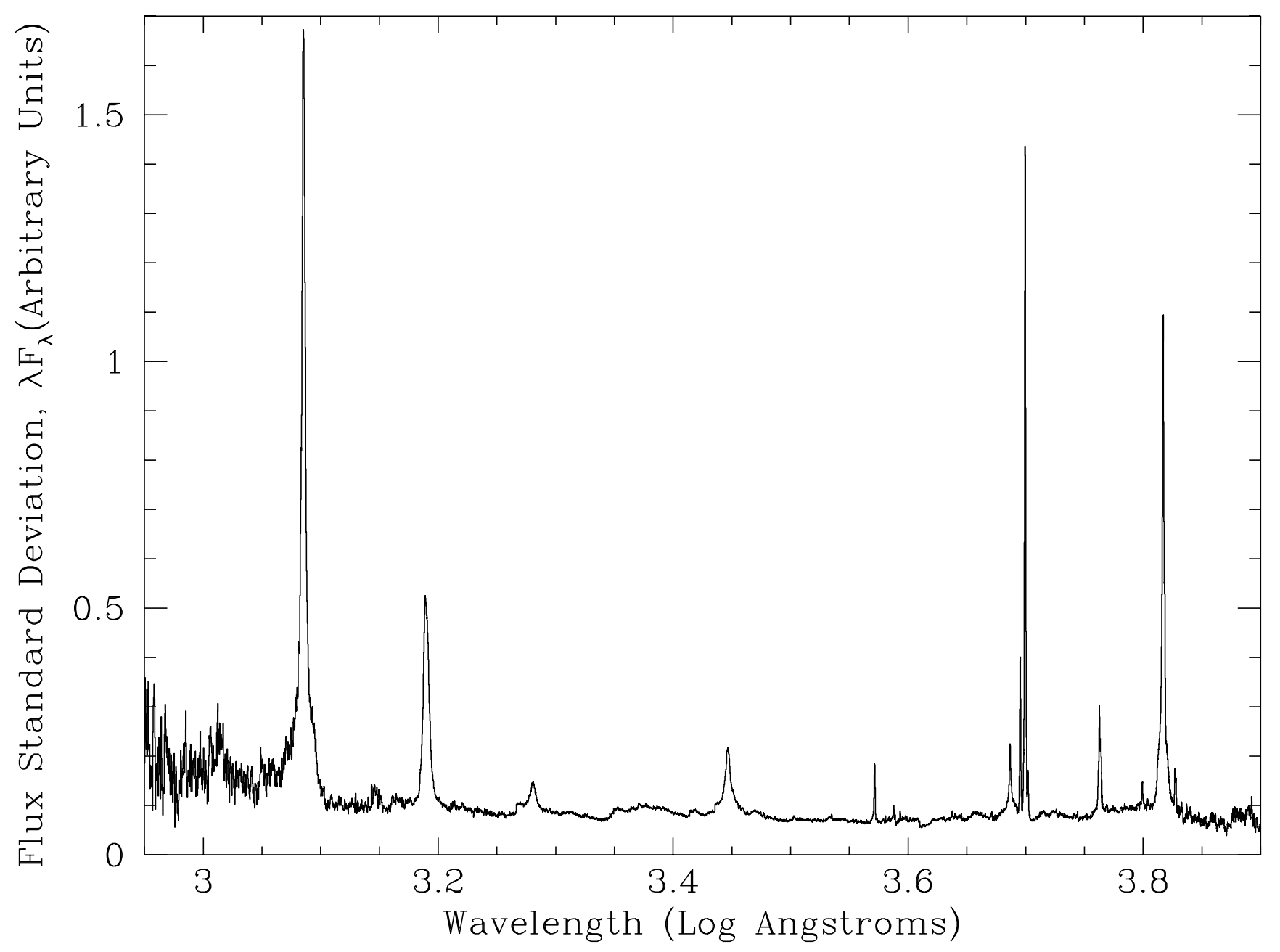

FIG. 3.- Standard deviation in $\lambda F_{\lambda}$ relative to the FBQS composite spectrum as a function of wavelength for the individual spectra comprising the composite. The same normalization constant has been used as in Fig. 1. 


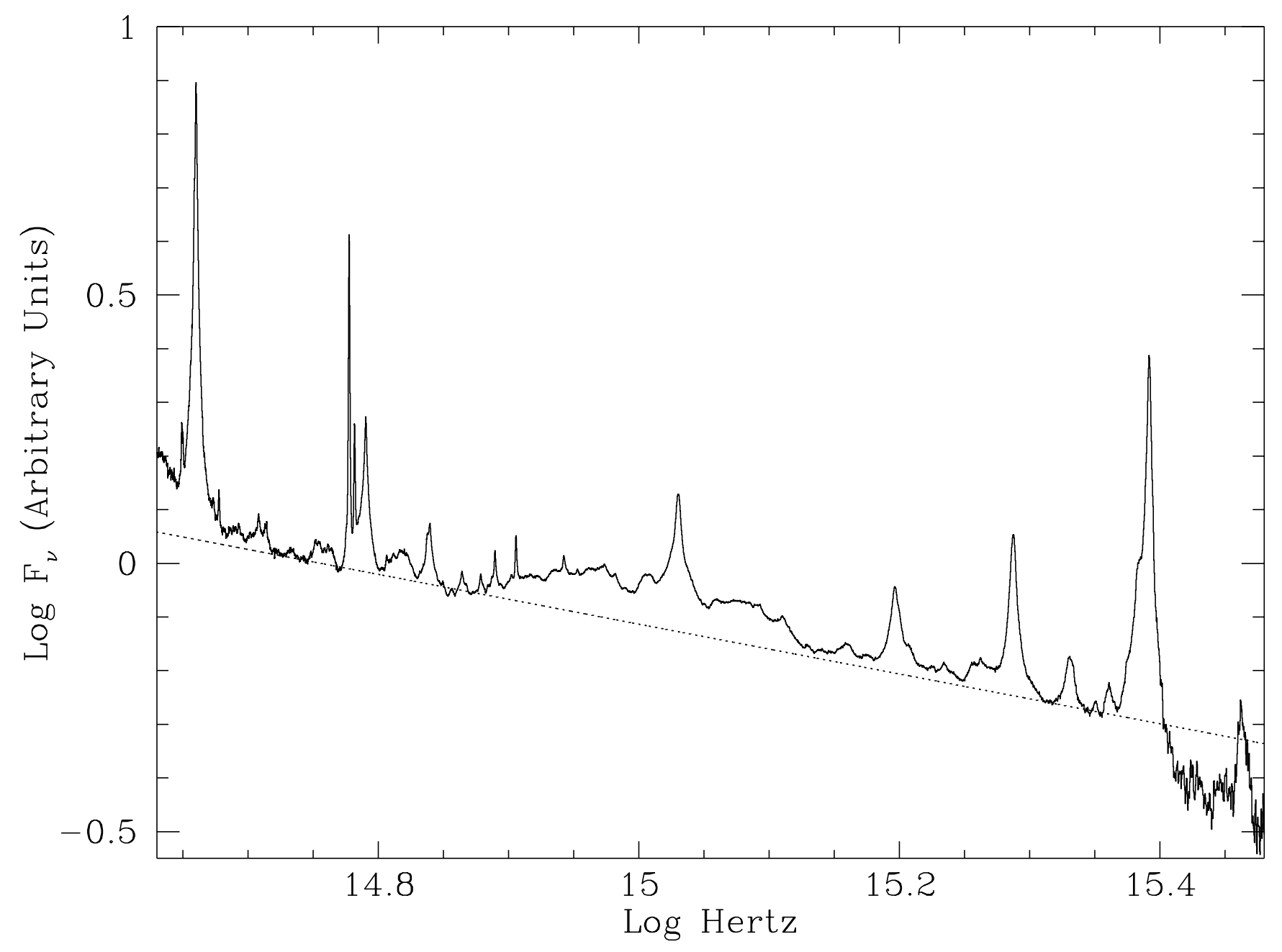

Fig. 4. - $\log \left(\mathrm{F}_{\nu}\right)-\log (\nu)$ plot of the FBQS composite (solid line) compared with a power law (dotted line) of index $\alpha=-0.46$ (where $\mathrm{F}_{\nu}$ $\left.\propto \nu^{\alpha}\right)$. 


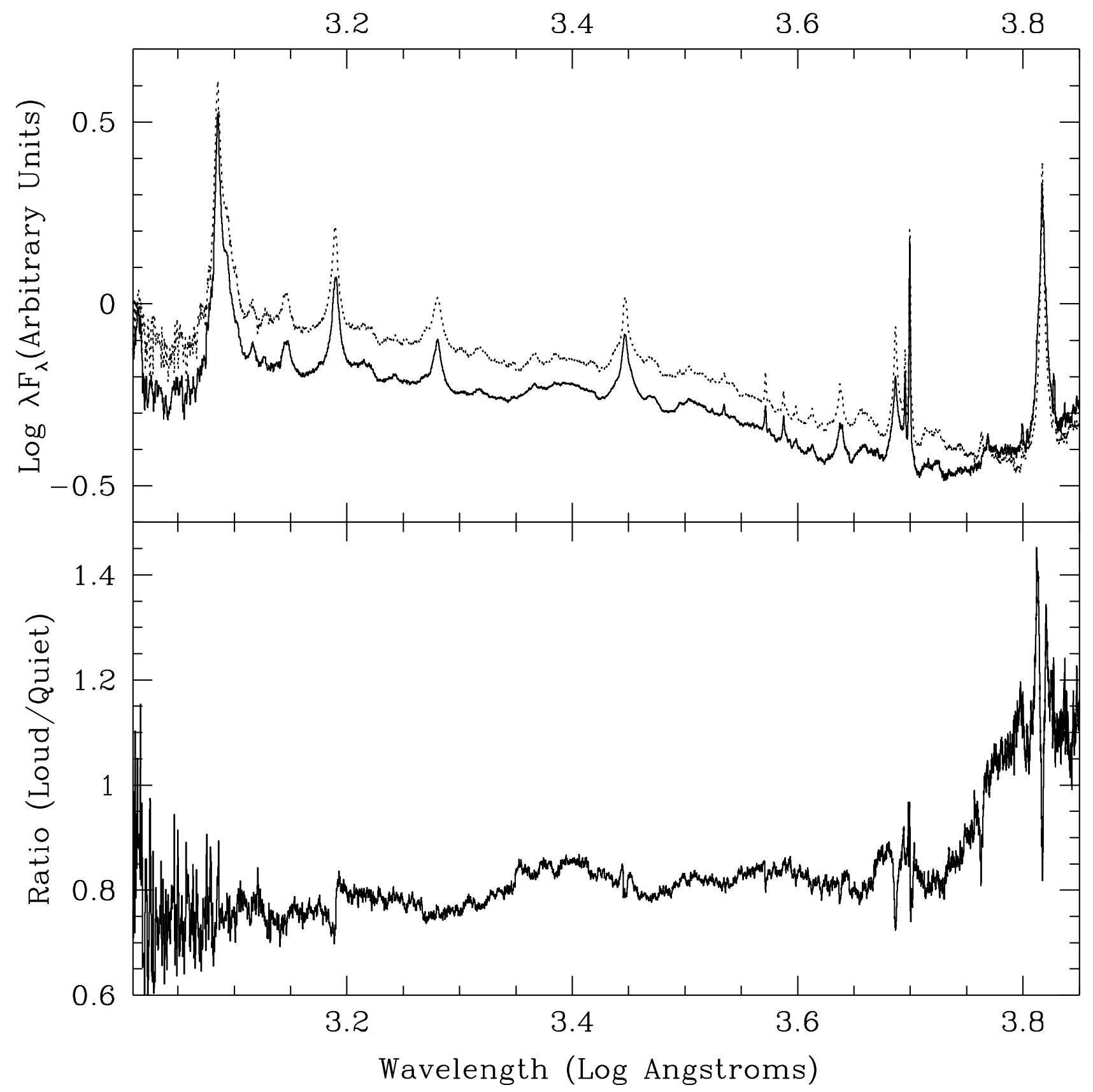

FIG. 5.- Upper panel. Comparison of mean spectra from the radio-loud (solid line) and radio-quiet (dotted line) subsamples of the FBQS. Bottom panel. Ratio of the radio-loud to radio-quiet composite spectrum. 


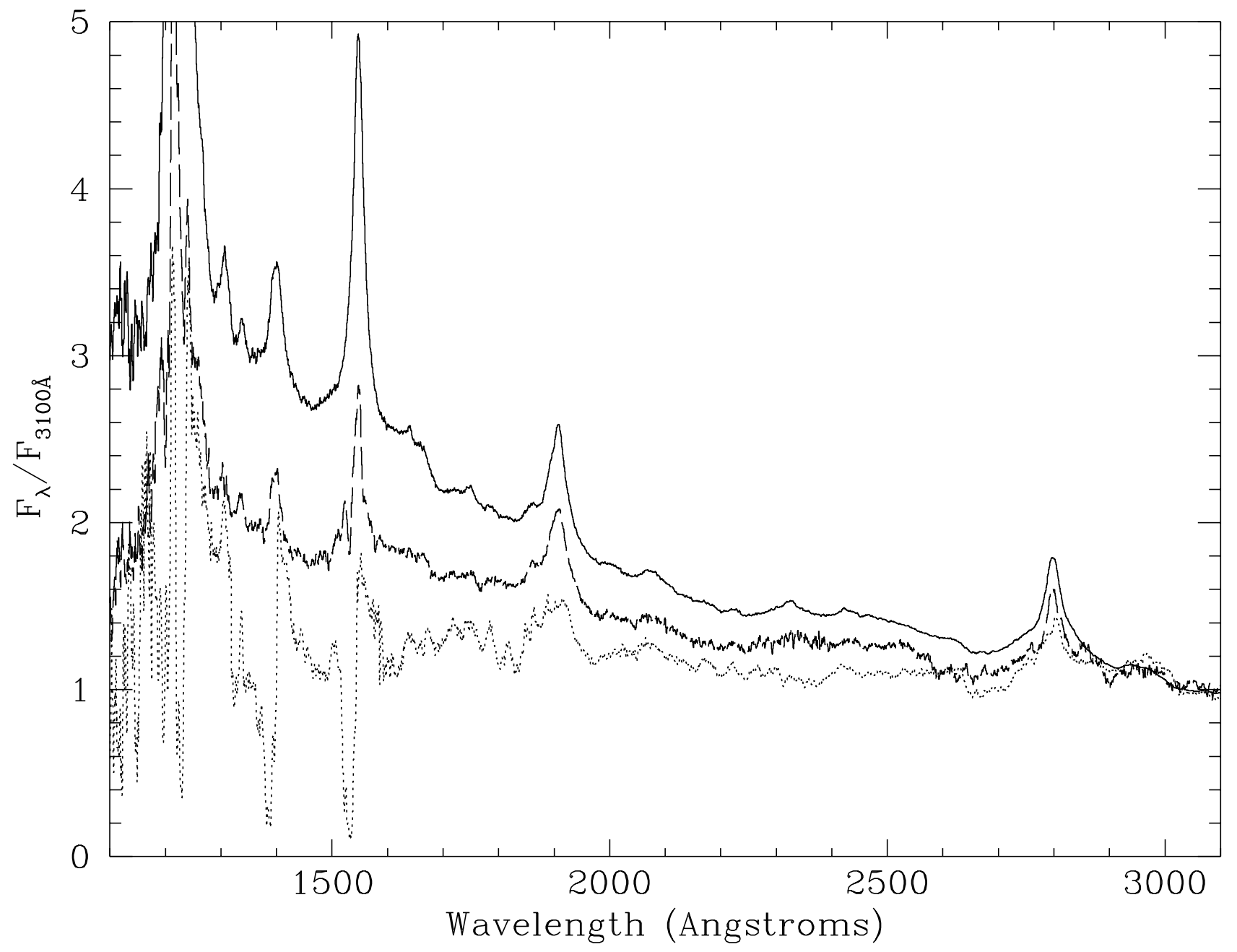

FIG. 6.- Comparison of mean spectra from the FBQS (solid line) and subsamples comprised of the high-ionization BAL quasars (dashed line) and low-ionization BAL quasars (dotted line). The spectra have been normalized to the flux at $3100 \AA$. 


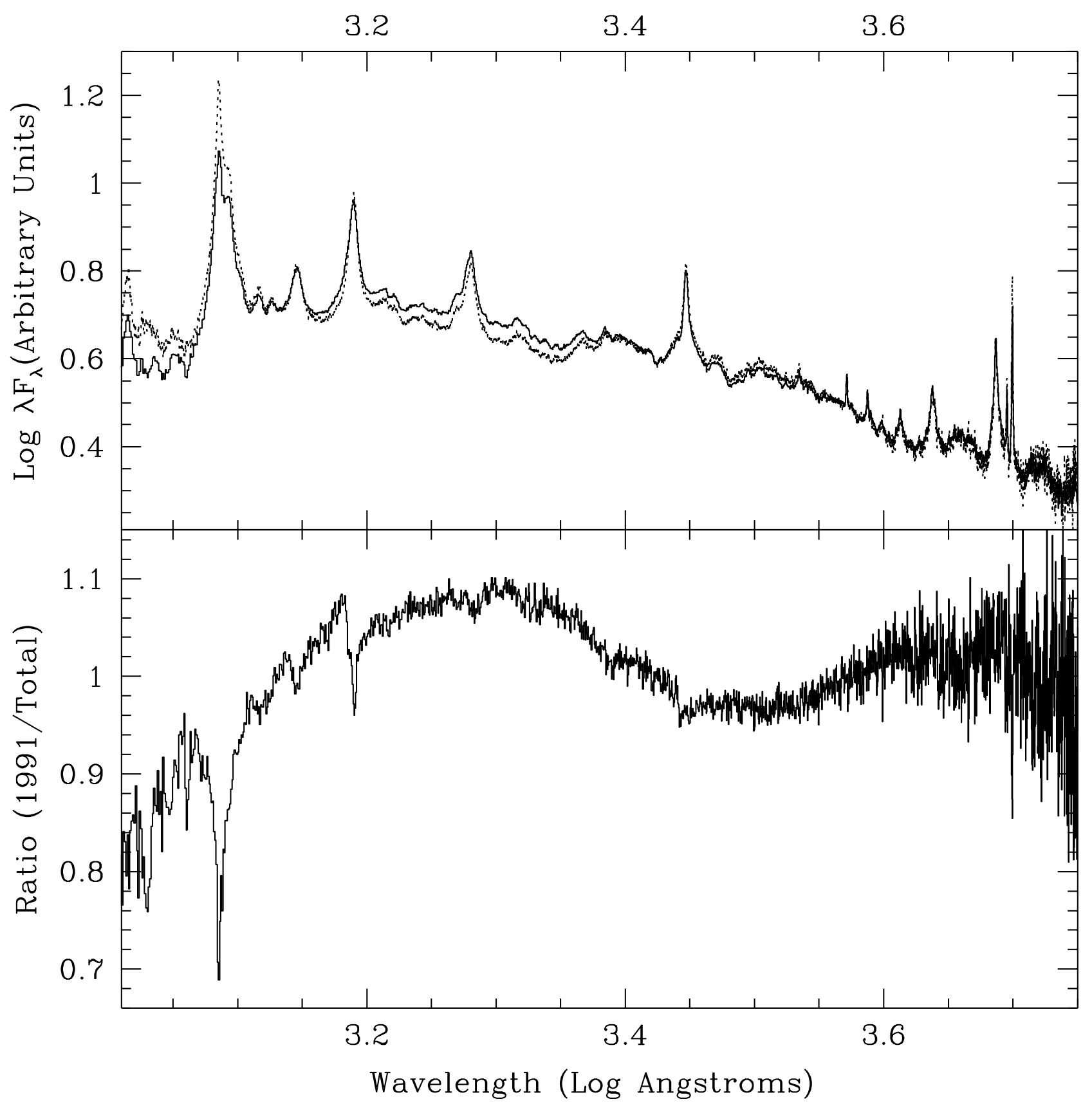

FIG. 7.- Upper panel. Comparison of mean spectra from the LBQS as presented by Francis et al. (1991) (solid line) and updated with the complete LBQS data set by Simon Morris (1999, private communication) (dotted line). Bottom panel. Ratio of published 1991 LBQS composite quasar spectrum to that created with the total sample. 


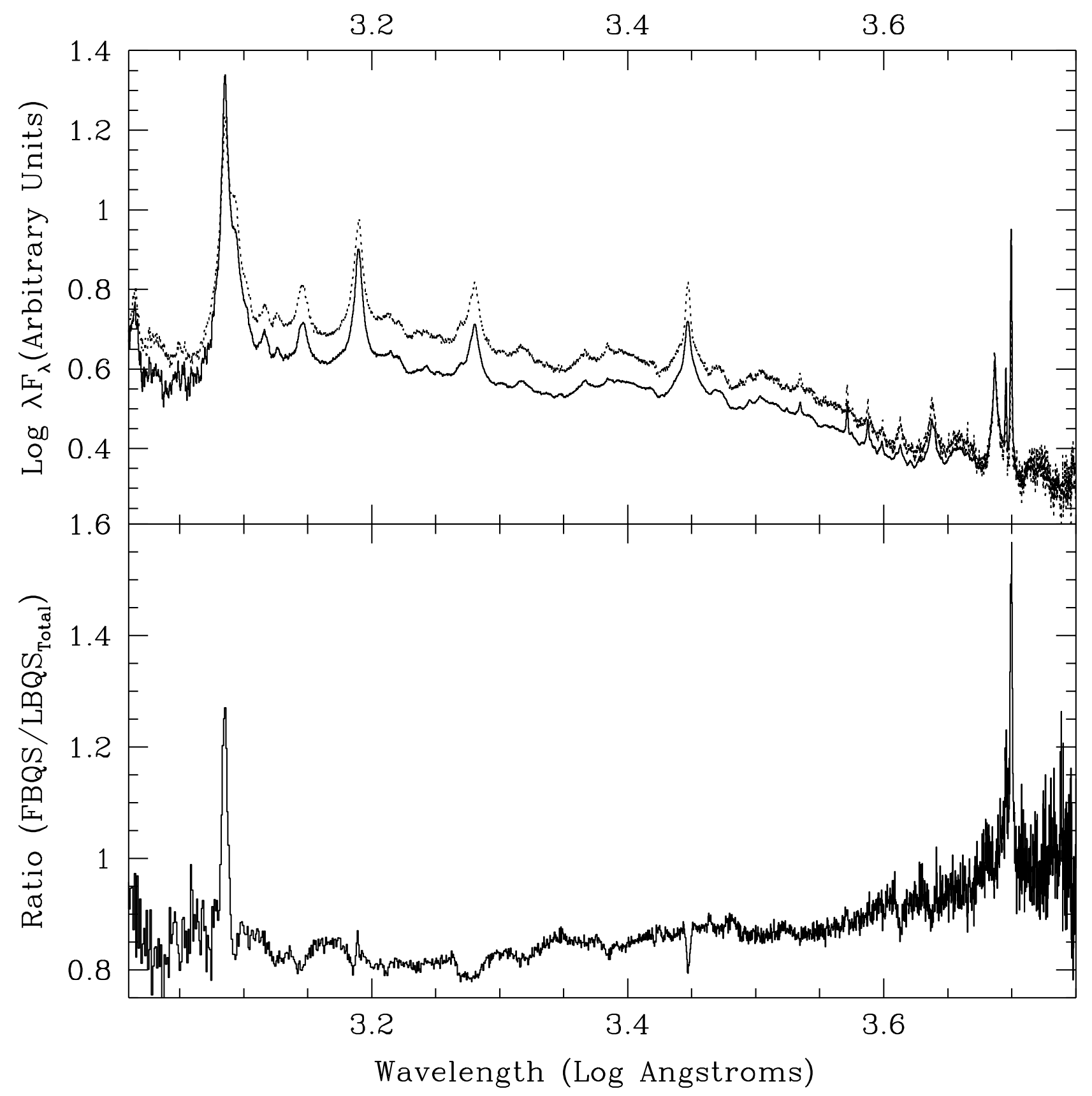

FIG. 8.- Upper panel. Comparison of mean spectra from the FBQS (solid line) and the total LBQS (dotted line). Bottom panel. Ratio of the FBQS composite quasar spectrum to that of the total LBQS sample. 